\title{
BARIUM STARS AND Tc-POOR S STARS: BINARY MASQUERADERS WITHIN THE CARBON-STAR FAMILY
}

\author{
A. JORISSEN AND S. VAN ECK \\ Institut d'Astronomie et d'Astrophysique, \\ Université Libre de Bruxelles, Belgium
}

Abstract. Our current understanding of the origin of barium and S stars is briefly reviewed, based on new orbital elements and binary frequencies.

\section{The relation of barium and $S$ stars to carbon stars}

Since the last conference devoted to chemically-peculiar red giant stars (IAU Coll. 106, Evolution of Peculiar Red Giant Stars, ed. Johnson \& Zuckerman, 1989), much progress has been made in understanding how barium and S stars relate to the other kinds of peculiar red giants. The discovery of the binary nature of barium stars (McClure et al. 1980; McClure 1983) suggested from the beginning that mass transfer was likely to play a key role in the formation of the barium syndrome. As far as S stars are concerned, it has become clear that Tc-rich and Tc-poor S stars form two separate families with similar chemical peculiarities albeit of very different origins (Iben \& Renzini 1983; Little et al. 1987; Jorissen \& Mayor 1988; Smith \& Lambert 1988; Brown et al. 1990; Johnson 1992; Jorissen \& Mayor 1992; Groenewegen 1993; Johnson et al. 1993; Jorissen et al. 1993; Ake, this conference). Tc-rich (or 'intrinsic') S stars are genuine thermally-pulsing AGB stars where the $s$-process operates in relation with the thermal pulses, and where the third dredge-up brings the freshly synthesized $s$-elements (including Tc) to the surface (e.g. Iben \& Renzini 1983; Sackmann \& Boothroyd 1991). By contrast, Tc-poor (or 'extrinsic') $S$ stars are believed to be the cool descendants of barium stars.

Figure 1 summarizes our current understanding of the relationship between the different families of peculiar red giant stars. This general picture raises several questions that will be addressed briefly in this paper:

- Is binarity a necessary condition to produce a barium star? 

1. main
$\circ \quad \circ$
$\underset{\text { sequence }}{\operatorname{main}}$
2. $M$
$\bigcirc \quad \circ$
3. $\mathrm{S}(\mathrm{Tc})$

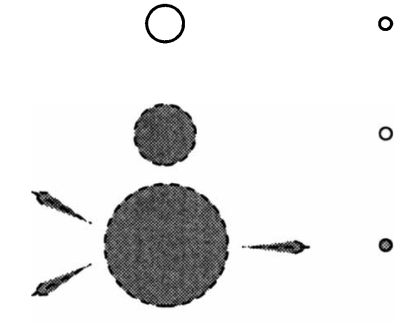
○
4. $\mathrm{C}(\mathrm{Tc})$

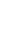
5. WD
-
Wind accretion
or RLOF
6.

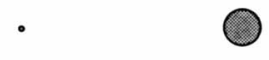
$\mathrm{Ba}$ (no Tc)
7.

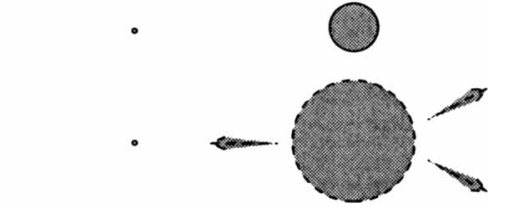
S(no Tc)
dwarf Ba or C
8.
$\mathrm{S}(\mathrm{Tc}), \mathrm{C}(\mathrm{Tc})$
9.
WD

Figure 1. Relationship between several families of peculiar red giant stars. Grey symbols represent heavy-element-rich stars, and dashed boundaries indicate Tc-rich stars. The left column depicts the normal (i.e. not requiring binarity) $\mathrm{M}-\mathrm{S}-\mathrm{C}$ evolution on the AGB, whereas the right column represents the evolution of a companion star. Note in particular the possibility that this companion itself evolves into a Tc-rich S star on the AGB, after having first shown up as a Tc-poor S star.

- What is the mass transfer mode [wind accretion or Roche lobe overflow (RLOF)] responsible for their formation?

- Do barium stars form as dwarfs or as giants?

- Do barium stars evolve into Tc-poor S stars?

- What is the relative frequency of Tc-rich and Tc-poor S stars?

- Are the abundances in the mass-loser star (i.e. the AGB progenitor of the present white dwarf companion) compatible with those presently observed in the barium or extrinsic S star?

We refer to Jorissen \& Boffin (1992), Han et al. (1995) and Busso et al. (1995) for a detailed discussion of the last item.

\section{Is binarity a necessary condition to form a barium star?}

To answer that question, barium stars with strong anomalies (including all $\mathrm{Ba} 4$ and $\mathrm{Ba} 5$ stars on the scale devised by Warner 1965 from the list of Lü et 
al. 1983) have been monitored either with the CORAVEL spectrovelocimeter (Baranne et al. 1979) or by McClure \& Woodsworth (1990). HD 19014 and HD 65854 are the only stars in that sample that do not show any sign of binary motion. For a fictitious population of binaries observed with the same time sampling and the same internal errors as the real sample of barium stars, and having eccentricity and mass-function distributions matching the observed ones, a Monte-Carlo simulation yields a binary detection rate of between $96 \%(35.4 / 37)$ and $98 \%(36.2 / 37)$, depending on whether the observed period distribution is extrapolated or not towards periods as long as $2 \times 10^{4} \mathrm{~d}$ [see Jorissen et al. 1998 for more details]. Binarity is thus a necessary condition to produce strong barium stars.

In a comparison sample of 40 mild barium stars (i.e. with $\mathrm{Ba} 1$ and $\mathrm{Ba} 2$ indices) randomly selected from the list of Lü et al. (1983) and monitored in a similar way as the strong barium stars, $34(85 \%)$ are definitely spectroscopic binaries, 3 (8\%) are probably binaries, and 3 (HD 50843, HD 95345, HD 119185) show no sign of radial velocity variations at the level of $0.3 \mathrm{~km} \mathrm{~s}^{-1}$ r.m.s. after more than 10 years of monitoring. Detailed spectroscopic abundance analyses performed on HD 95345 (Sneden et al. 1981) and HD 119185 (Začs et al. 1997) confirm the existence of mild heavy-element overabundances $([s / \mathrm{Fe}]=0.2$ to $0.3 \mathrm{dex})$ for these stars with constant radial velocity. This frequency of constant stars is again consistent with the binary detection rate predicted for that sample by a Monte-Carlo simulation, provided that the period distribution of mild barium stars extends up to $2 \times 10^{4} \mathrm{~d}$. In these conditions, there is no need to invoke any formation mechanism other than mass transfer in a binary system to produce mild barium stars. On the contrary, an alternative formation scenario (such as galactic fluctuations of the $s /$ Fe ratio; Williams 1975, Sneden et al. 1981, Edvardsson et al. 1993) may be required to account for a population of non-binary stars found among dwarf mild barium stars (North et al., this conference).

Is binarity a sufficient condition to produce a barium star? Probably not, since binary systems consisting of a normal red giant and a WD companion with Ba-like orbital parameters do exist (Jorissen \& Boffin 1992). DR Dra (= HD 160538) is probably the best example, with $P=904 \mathrm{~d}$, $e=0.07$ (compare with Fig. 3 ) and a hot WD companion detected by Fekel et al. (1993). Berdyugina (1994) finds a metallicity close to solar and normal $\mathrm{Zr}$ and La abundances in the giant. Začs et al. (1997) basically confirm that result.

Metallicity may be the other key parameter, besides binarity, controlling the formation of barium stars. The $s$-process efficiency, expressed in terms of the neutron irradiation, seems to be larger in low-metallicity stars (Kovács 1985; Busso et al. 1995). Clayton (1988) provides a theoretical foundation 


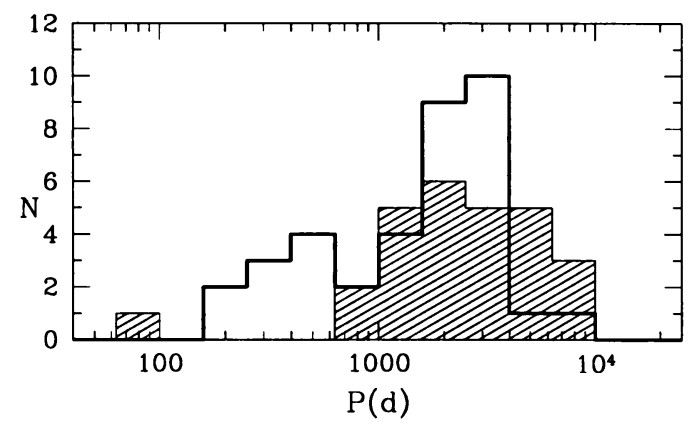

Figure 2. The distribution of orbital periods for mild barium stars (shaded histogram) and strong barium stars (thick line) (from McClure \& Woodsworth 1990 and Jorissen et al. 1998). The distribution is complete up to about $4000 \mathrm{~d}$.

for that empirical finding, provided that ${ }^{13} \mathrm{C}(\alpha, \mathrm{n}){ }^{16} \mathrm{O}$ is the neutron source for the $s$-process. Barium stars would therefore be easier to produce in a low-metallicity population.

\section{Inferring the mass transfer mode from the orbital elements: Wind accretion and/or Roche lobe overflow?}

Synthetic binary evolution models (Han et al. 1995; de Kool \& Green 1995) suggest that the bimodal period distribution exhibited by strong barium stars (Fig. 2) reflects the operation of two distinct mass-transfer modes, RLOF in the short-period mode (peaking around $500 \mathrm{~d}$ ) and wind accretion in the long-period mode (around $3000 \mathrm{~d}$ ).

This general picture actually faces three major difficulties: first, the threshold period (about $1000 \mathrm{~d}$ ) between the RLOF and wind-accretion modes is much too short to accomodate the large radii reached by AGB stars. Second, the period - eccentricity diagram (Fig. 3) reveals that not all orbits in the short-period (i.e. post-RLOF) mode are circular, although tidal effects are expected to circularize the orbit in the phase of large radius just preceding RLOF (e.g. Zahn 1977). A similar problem exists for the orbits of dwarf barium stars (see North et al., this conference). Third, RLOF from AGB stars with a deep convective envelope is dynamically unstable ('unstable case C RLOF'; e.g. Tout \& Hall 1991), with the ensuing common envelope stage generally accompanied by dramatic orbital shrinkage leading to the formation of a cataclysmic binary with a period much shorter than that of barium stars (e.g. Meyer \& Meyer-Hofmeister 1979). To solve these problems, Han et al. (1995), Livio (1996) and Jorissen et al. (1998) propose avenues to explore. One of these involves Eggleton's CRAP (CompanionReinforced Attrition Process; Eggleton 1986), speculating that larger massloss rates for AGB stars in binary systems may reverse the mass ratio of the system prior to RLOF, thus stabilizing the mass transfer process (Tout 


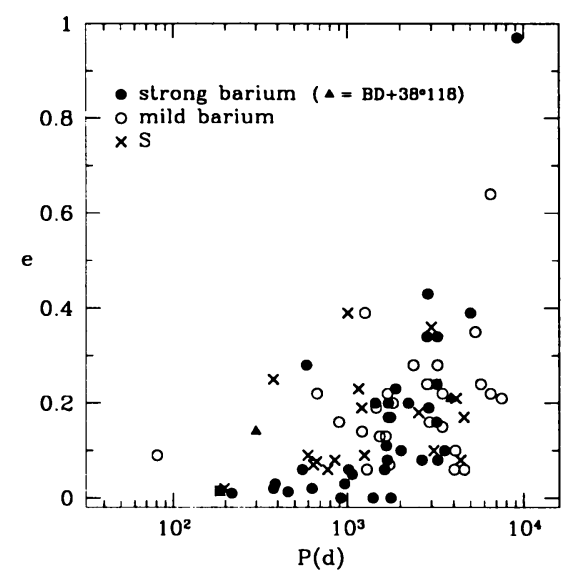

Figure 3. The $(e, \log P)$ diagram for barium and S stars (from Jorissen et al. 1998). $\mathrm{BD}+38^{\circ} 118$ is a triple hierarchical system, with the close inner binary and the orbit of the third star around the center of mass of the inner binary represented by filled triangles.

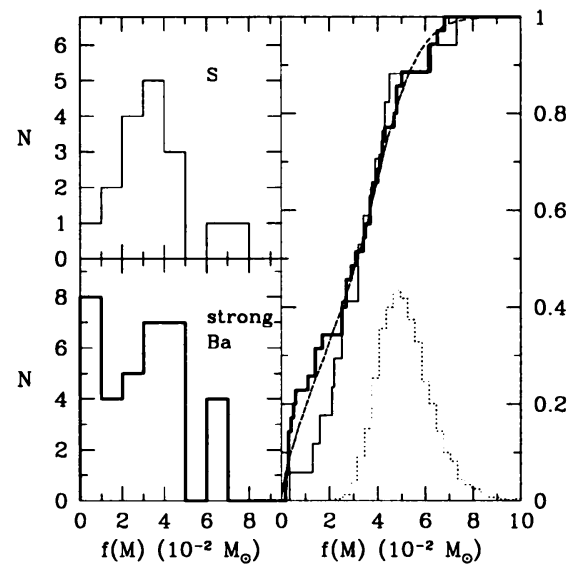

Figure 4. The mass-function distributions of strong barium stars (thick line) and $\mathrm{S}$ stars (thin line) from Jorissen et al. (1998), excluding the peculiar S systems HDE 332077 and HD 191589 (see text). The dashed line in the right panel is the fit obtained with the $Q$ distribution shown (dotted line).

\& Eggleton 1988; Han et al. 1995).

\section{Do barium stars form as dwarfs or as giants?}

In Fig. 1, it is assumed that the mass transfer responsible for the barium syndrome occurred when the barium star was still on the main sequence. Because the stellar lifetime is longer on the main sequence than in the giant phase, that possibility indeed appears more probable than the formation of the barium star directly as a giant star. However, as pointed out by Iben \& Tutukov (1985), the mismatch between the thermal time scale of the dwarf's envelope and that of the mass-losing AGB star may prevent the formation of dwarf barium stars. A main-sequence star would indeed be driven out of thermal equilibrium in case of rapid mass accretion from its giant companion, and would swell to giant dimensions (e.g. Kippenhahn \& Meyer-Hofmeister 1977), leading to a common envelope stage with possibly dramatic consequences on the fate of the binary system (see e.g. Meyer \& Meyer-Hofmeister 1979, and Sect. 3). Dwarf barium stars long remained elusive, until Luck \& Bond $(1982,1991)$ and North et al. (1994) recognized that some of the $\mathrm{CH}$ subgiants previously identified by Bond (1974), as well as some of the F dwarfs previously classified by Bidelman (1985) as having 'strong $\operatorname{Sr} \lambda$ 4077', had the proper abundance anomalies, gravities 
and galactic frequencies to be identified with the long-sought $\mathrm{Ba}$ dwarfs. A large fraction of binaries (about $90 \%$ ) has been found among the stars with strong anomalies, as expected (McClure 1985; North \& Duquennoy 1992; North et al., this conference). The very existence of binary dwarf $\mathrm{Ba}$ stars, in spite of Iben \& Tutukov's argument, is another indication that, if RLOF does indeed occur in these systems, it does not have the catastrophic consequences generally associated with unstable case C RLOF. The question of whether these dwarf barium stars will eventually evolve into giant barium stars is addressed by North et al. in these Proceedings.

The formation of a barium star directly as a giant, though probably less frequent, is by no means excluded. The barium star HD 165141 may be such a case. HD 165141 is unique in sharing properties of barium and RS CVn systems (Fekel et al. 1993; Jorissen et al. 1996). Its rapid rotation $(V \sin i=$ $14 \mathrm{~km} \mathrm{~s}^{-1}$ ) and X-ray flux (probably from a hot corona) are typical of RS CVn systems. However, the spin-up of that star (and the concomittant RS CVn properties) cannot be attributed to tidal effects synchronizing the stellar rotation with the orbit, as is the case for RS CVn systems, since the orbital period (about $5200 \mathrm{~d}$ ) is much too long. That puzzle may be solved if the wind accretion episode responsible for the barium syndrome spun the star up, as suggested by detailed hydrodynamical simulations (Theuns \& Jorissen 1993; Theuns et al. 1996). Since magnetic braking is generally faster than the stellar lifetime on the giant branch, wind accretion and concomittant spin-up must have occurred when HD 165141 was already a giant star. Strong support for that hypothesis comes from the fact that HD 165141 has a hot WD companion (Fekel et al. 1993) whose cooling time scale is shorter than the lifetime of HD 165141 on the red giant branch. Finally, note that Jeffries \& Stevens (1996) have reported more cases of WIRRing (Wind-Induced Rapidly Rotating) stars among binaries involving a hot WD.

\section{Do barium stars evolve into Tc-poor $S$ stars?}

Figure 3 shows that strong barium stars and Tc-poor S stars occupy the same region of the $(e, \log P)$ diagram. The distributions of the mass function $f(M)$ presented in Fig. 4 [where $f(M)=M_{2}^{3} \sin ^{3} i /\left(M_{1}+M_{2}\right)^{2} \equiv$ $Q \sin ^{3} i, M_{1}$ and $M_{2}$ being the masses of the giant and of the WD, respectively] for the two families are compatible with the hypothesis that they are extracted from the same parent population. Following the usual analysis (Webbink 1986; McClure \& Woodsworth 1990) of the mass function distribution in terms of a peaked distribution of mass ratios $Q$ convolved with randomly inclined orbits, an average ratio $Q=0.045 M_{\odot}$ is found for the two classes, translating into a giant mass of $1.6 M_{\odot}$ when adopt- 
ing $M_{2}=0.6 M_{\odot}$ for the WD companion. These two results thus provide strong support for the hypothesis that strong barium and Tc-poor S stars represent successive stages in the evolutionary path sketched in Fig. 1.

Note, however, that the above comparison of the mass functions does not include two Tc-poor S stars (HD 191589 and HDE 332077) with main sequence companions detected with the International Ultraviolet Explorer satellite (Ake \& Johnson 1992; Ake et al. 1992). The evolutionary status of these stars is currently unknown.

\section{The relative frequency of intrinsic/extrinsic $\mathbf{S}$ stars}

The evaluation of the relative frequency of intrinsic/extrinsic $S$ stars faces two difficulties: $(i)$ one needs an efficient criterion for distinguishing extrinsic from intrinsic $\mathrm{S}$ stars, and (ii) the frequency evaluation must be corrected for selection bias, since extrinsic and intrinsic S stars follow different galactic distributions (Jorissen et al. 1993). As far as $(i)$ is concerned, the defining criterion of intrinsic/extrinsic $S$ stars based on the presence/absence of Tc, respectively, may be difficult to apply to a complete sample of S stars such as Henize's (see below), since it involves many faint stars for which high-resolution spectroscopy is difficult to secure. Binarity may be an alternative, since the binary paradigm for $\mathrm{S}$ stars states that all Tc-poor S stars should be binaries (Brown et al. 1990; Johnson 1992). However, some binaries must be expected among Tc-rich $S$ stars as well, as in any class of stars. Binary intrinsic S stars with main sequence companions (case 3 in Fig. 1) include the close visual binary $\pi^{1}$ Gru (Feast 1953) and stars with composite spectra like T Sgr, W Aql, WY Cas (Herbig 1966; Culver \& Ianna 1975), and possibly S Lyr (Merrill 1956). The situation is further confused by extrinsic $S$ stars reaching the AGB phase and eventually becoming Tc-rich (case 8 in Fig. 1). $o^{1}$ Ori, a Tc-rich binary S star with a WD companion (Ake \& Johnson 1988), may be such a case.

The CORAVEL $S b$ parameter, measuring the average line width (see Jorissen \& Mayor 1988 for a more detailed definition), offers an interesting and efficient alternative to identify extrinsic/intrinsic S stars. In cool red giants where macroturbulence is the main line-broadening factor, the $S b$ parameter may be expected to be a sensitive function of the luminosity, as is macroturbulence (e.g. Gray 1988). But at the same time, bright giants exhibit large velocity jitters probably caused by envelope pulsations (e.g. Mayor et al. 1984). A correlation between $S b$ and the radial velocity jitter must thus be expected, as observed in Fig. 5 for barium, intrinsic and extrinsic S stars (Jorissen \& Mayor 1992; Jorissen et al. 1998).

All Tc-poor S stars are binary stars, as expected, but moreover, they are restricted to $S b<5 \mathrm{~km} \mathrm{~s}^{-1}$. That criterion has been used to identify 


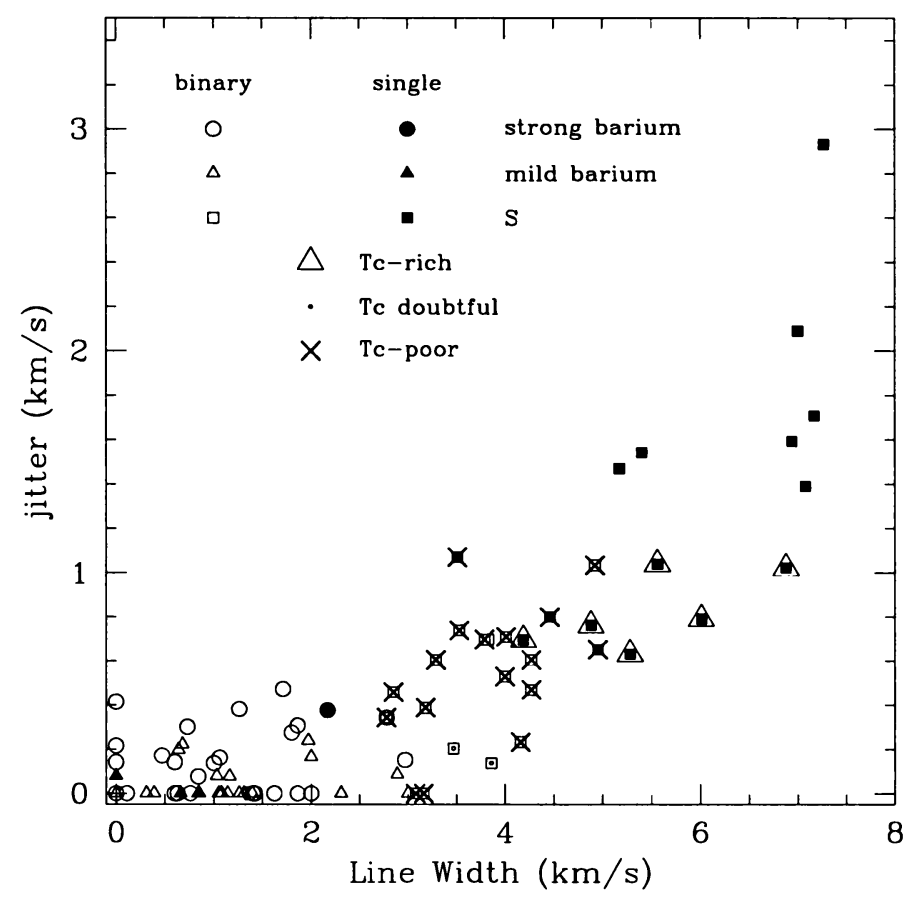

Figure 5. The jitter $\left(\sigma^{2}-\bar{\epsilon}_{1}^{2}\right)^{1 / 2}$ (where $\bar{\epsilon}_{1}$ is the average error on one measurement, and $\sigma$ is the standard deviation of the radial velocity for single stars, and of the $O-C$ residuals around the computed orbit for binary stars) vs. the CORAVEL line-width parameter $S b$ (see text).

intrinsic S stars among the Henize sample (Henize 1960). That sample covers the sky south of declination $-25^{\circ}$ uniformly to red magnitude 10.5 , and $205 \mathrm{~S}$ stars were found from their $\mathrm{ZrO} \lambda 6345$ band on red-yellow spectra with a dispersion of $450 \AA \mathrm{mm}^{-1}$ at $\mathrm{H} \alpha$. The galactic distribution of the Henize sample is presented in Fig. 6. Intrinsic S stars are clearly more concentrated towards the galactic plane than extrinsic S stars. Correcting for the uneven sampling of galactic latitudes, the frequency of intrinsic $\mathrm{S}$ stars (based on the $S b>5 \mathrm{~km} \mathrm{~s}^{-1}$ criterion) then amounts to at least $62 \pm 5 \%$ (in a magnitude-limited sample).

It is our pleasure to thank M. Mayor and the CORAVEL team at the Observatoire de Genève for making possible the long-term radial-velocity monitoring discussed here. A.J. is Research Associate, Fonds National de la Recherche Scientifique (Belgium); S.V.E. is Boursier F.R.I.A. (Belgium). We thank the Fonds National de la Recherche Scientifique (Belgium), the Communauté Française de Belgique and the symposium Organizing Committee for financial support. 


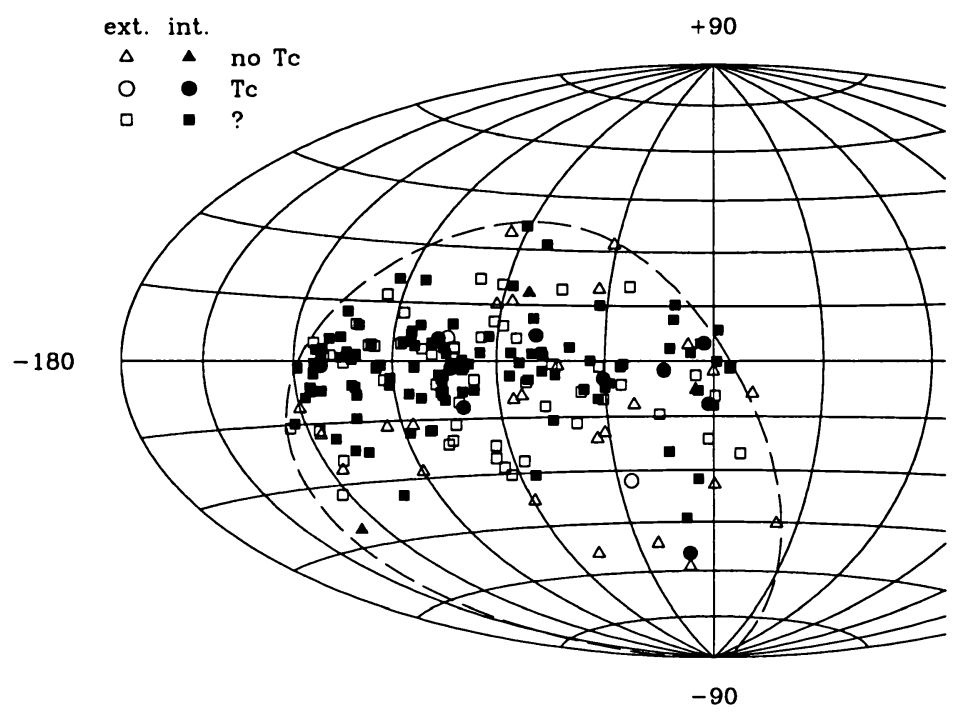

Figure 6. Galactic distribution of S stars from the Henize sample.

\section{References}

Ake, T. B. \& Johnson, H. R. 1988, ApJ, 327, 214

Ake, T. B. \& Johnson, H. R. 1992, in Cool Stars, Stellar Systems and the Sun, ed. M.S. Giampapa and J. A. Bookbinder, ASP Conf. Ser., 26, 579

Ake, T., Jorissen, A., Johnson, H., Mayor, M. \& Bopp, B. 1992, Bull. Am. Astron. Soc., 24,1280

Baranne, A., Mayor, M. \& Poncet, J. L. 1979, Vistas in Astronomy, 23, 279

Berdyugina, S. V. 1994, Pi'sma Astron. J., 20, 910

Bidelman, W. P. 1985, $A J, 90,341$

Bond, H.E. 1974, ApJ, 194, 95

Brown, J. A., Smith, V.V., Lambert, D. L., Dutchover, E. Jr., Hinkle, K. H. \& Johnson, H. R. $1990, A J, 99,1930$

Busso, M., Lambert, D. L., Beglio, L., Gallino, R., Raiteri, C. M. \& Smith, V. V. 1995, ApJ, 446, 775

Clayton, D. D. 1988, MNRAS, 234, 1

Culver, R. B. \& Ianna, P. A. $1975, A p J, 195$, L37

de Kool, M. \& Green, P. J. 1995, ApJ, 449, 236

Edvardsson, B., Andersen, J., Gustafsson, B., Lambert, D. L., Nissen, P.E. \& Tomkin, J. 1993, $A \& A, 275,101$

Eggleton, P.P. 1986, in The Evolution of Galactic X-ray Binaries, ed. J. Trümper, W. H. G. Lewin and W. Brinkmann (Reidel), p. 87

Feast, M.W. 1953, MNRAS, 113, 510

Fekel, F. C., Henry, G. W., Busby, M. R. \& Eitter, J. J. 1993, AJ, 106, 2370

Gray, R.F. 1988, Lectures on Spectral-line Analysis: F, G and $K$ stars, (Arva, Ontario: The Publisher)

Groenewegen, M. A. T. 1993, $A \mathscr{E} A, 271,180$

Han, Z., Eggleton, P.P., Podsiadlowski, P. \& Tout, C. A. 1995, MNRAS, 277, 1443 
Henize, K. G. 1960, $A J, 65,491$

Herbig, G. H. 1966, $A J, 71,779$

Iben, I. Jr. \& Renzini, A. 1983, Ann. Rev. Astron. Astrophys., 21, 271

Iben, I. Jr. \& Tutukov, A. V. 1985, ApJ Supp., 58, 661

Jeffries, R. D. \& Stevens, I. R. 1996, MNRAS, 279, 180

Johnson, H. R. 1992, in IAU Symp. 151: Evolutionary Processes in Interacting Binary Stars, ed. Y. Kondo, R. F. Sistero, and R. S. Polidan (Kluwer), p. 157

Johnson, H. R., Ake, T.B. \& Ameen, M. M. 1993, ApJ, 402, 667

Jorissen, A. \& Boffin, H. M. J. 1992, in Binaries as Tracers of Stellar Formation, ed. A. Duquennoy and M. Mayor (Cambridge), p. 110

Jorissen, A., Frayer, D. T., Johnson, H. R., Mayor, M. \& Smith, V. V. 1993, $A \& A$, 271, 463

Jorissen, A. \& Mayor, M. 1988, $A \& A, 198,187$

Jorissen, A. \& Mayor, M. 1992, $A \& A, 260,115$

Jorissen, A., Schmitt, J. H. M. M., Carquillat, J. M., Ginestet, N. \& Bickert, K. F. 1996, $A \mathcal{E} A, 306,467$

Jorissen, A., Van Eck, S., Mayor, M. \& Udry, S. 1998, $A \& A, 332,877$

Kippenhahn, R. \& Meyer-Hofmeister, E. 1977, $A \& A, 54,539$

Kovács, N. 1985, $A \& A, 150,232$

Little, S. J., Little-Marenin, I. R. \& Bauer, W. H. 1987, $A J, 94,981$

Livio, M. 1996, in Evolutionary Processes in Binary Stars, ed. R. A. M. J. Wijers, M. B. Davies, and C. A. Tout (Kluwer), p. 141

Lü, P. K., Dawson, D. W., Upgren, A.R. \& Weis, E.W. 1983, ApJ Supp., 52, 169

Luck, R.E. \& Bond, H.E. 1982, ApJ, 259, 792

Luck, R. E. \& Bond, H.E. 1991, ApJ Supp., 77, 515

Mayor, M., Imbert, M., Andersen, J., Ardeberg, A., Benz, W., Lindgren, H., Martin, N., Maurice, E., Nordström, B. \& Prévot, L. 1984, $A \& A, 134,118$

McClure, R. D. 1983, ApJ, 268, 264

McClure, R. D. 1985, in Cool Stars with Excesses of Heavy Elements, ed. M. Jaschek and P. C. Keenan (Reidel), p. 159

McClure, R. D., Fletcher, J. M. \& Nemec, J. M. 1980, ApJ, 238, L35

McClure, R. D. \& Woodsworth, A.W. 1990, ApJ, 352, 709

Merrill, P. W. 1956, PASP, 68, 162

Meyer, F. \& Meyer-Hofmeister, E. 1979, $A \& A, 78,167$

North, P., Berthet, S. \& Lanz, T. 1994, $A \& A, 281,775$

North, P. \& Duquennoy, A. 1992, in Binaries as Tracers of Stellar Formation, ed. A. Duquennoy and M. Mayor (Cambridge), p. 202

Sackmann, I.-J. \& Boothroyd, A. I. 1991, in IAU Symp. 145: Evolution of Stars: The Photospheric Abundance Connection, ed. G. Michaud and A. Tutukov (Kluwer), p. 275

Smith, V. V. \& Lambert, D. L. 1988, ApJ, 333, 219

Sneden, C., Lambert, D. L. \& Pilachowski, C. A. 1981, ApJ, 247, 1052

Theuns, T., Boffin, H. M. J. \& Jorissen, A. 1996, MNRAS, 280, 1264

Theuns, T. \& Jorissen, A. 1993, MNRAS, 265, 946

Tout, C. A. \& Eggleton, P. P. 1988, MNRAS, 231, 823

Tout, C. A. \& Hall, D.S. 1991, MNRAS, 253, 9

Warner, B. 1965, MNRAS, 129, 263

Webbink, R.F. 1986, in Critical Observations vs. Physical Models for Close Binary Systems, ed. K. C. Leung and D. S. Zhai (Gordon and Breach), p. 403

Williams, P. M. 1975, MNRAS, 170, 343

Začs, L., Musaev, F.A., Bikmaev, I.F. \& Alksnis, O. 1997, A\&A Supp., 122, 31

Zahn, J.-P. 1977, $A \& A, 57,383$ 